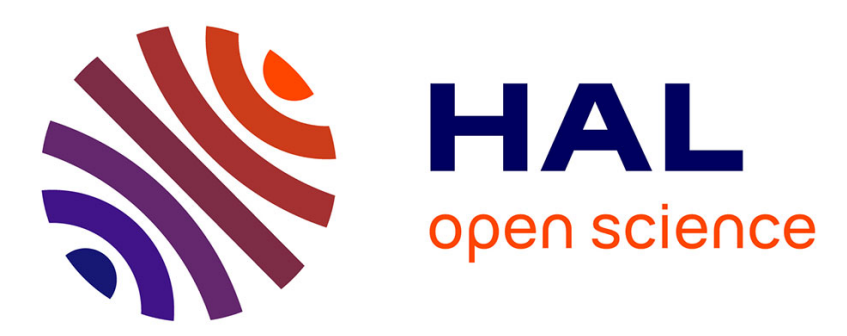

\title{
Adaptation d'une détection photoélectrique sur un spectrographe
}

\author{
C. Wecker, J.G. Gies, J.C. Merle, P. Fiorini
}

\section{To cite this version:}

C. Wecker, J.G. Gies, J.C. Merle, P. Fiorini. Adaptation d'une détection photoélectrique sur un spectrographe. Revue de Physique Appliquée, 1979, 14 (8), pp.799-800. 10.1051/rphysap:01979001408079900 . jpa-00244663

\section{HAL Id: jpa-00244663 https://hal.science/jpa-00244663}

Submitted on 1 Jan 1979

HAL is a multi-disciplinary open access archive for the deposit and dissemination of scientific research documents, whether they are published or not. The documents may come from teaching and research institutions in France or abroad, or from public or private research centers.
L'archive ouverte pluridisciplinaire HAL, est destinée au dépôt et à la diffusion de documents scientifiques de niveau recherche, publiés ou non, émanant des établissements d'enseignement et de recherche français ou étrangers, des laboratoires publics ou privés. 


\title{
Adaptation d'une détection photoélectrique sur un spectrographe
}

\author{
C. Wecker, J. G. Gies, J. C. Merle et P. Fiorini $\left(^{*}\right)$ \\ Laboratoire de Spectroscopie et d'Optique du Corps Solide, Associé au C.N.R.S. n 232, \\ Université Louis-Pasteur, 5, rue de l'Université, 67000 Strasbourg, France \\ (*) Istituto di Fisica, Università di Roma, Italie \\ (Reçu le 1er février 1979, révisé le 27 avril 1979, accepté le 4 mai 1979)
} Résumé. - Nous décrivons une détection photoélectrique amovible qui prend la place du châssis porte-plaque
d'un spectrographe. La résolution optimale est de $0,035 \AA$, la reproductibilité des mesures est de $0,01 \AA$.

Abstract. - We describe a removable photoelectric detection which takes place of the photographic plate holder of a spectrograph. The best resolution is $0.035 \AA$ and the reproductibility of measurements is $0.01 \AA$.

Les dispositifs dispersifs, destinés à l'étude des spectres optiques, utilisent deux modes de détection, soit photographique, soit photoélectrique. Quelques applications exigent les avantages complémentaires de chacune de ces détections et certains appareils permettent leur emploi. Nous disposions déjà d'un spectrographe performant, de marque Bausch et Lomb, disposant d'un réseau de 2160 traits $/ \mathrm{mm}$. Sa distance focale étant de $2 \mathrm{~m}$, la dispersion linéaire atteint $2 \AA / \mathrm{mm}$ au voisinage de $4000 \AA$. Le principe de sa transformation en spectrophotomètre, décrite ciaprès, a l'avantage d'être simple et applicable à la plupart des spectrographes.

A la place du châssis porte-plaque amovible, nous introduisons un autre châssis comportant un ensemble d'analyse constitué par une fente mobile suivie d'un tube photomultiplicateur qui recueille la lumière issue de cette dernière. La fente dispose de tous les réglages nécessaires à sa coïncidence avec l'image de la fente d'entrée du spectrographe (mise au point, largeur et inclinaison). Cet ensemble d'analyse est translaté le long du spectre au moyen d'une vis micrométrique dont la rotation est commandée par un moteur synchrone à travers une boîte de vitesse. Un système de repérage est directement associé à la rotation de la vis micrométrique; il donne un signal électrique chaque fois que la vis a tourné de $1 / 20$ de tour. Le pas de la vis étant de $1 \mathrm{~mm}$ et la dispersion linéaire de $2 \AA / \mathrm{mm}$, le repérage des longueurs d'onde est obtenu tous les $0,1 \AA$.

Les performances de cette détection photoélectrique sont les suivantes :

i) La course de l'ensemble mobile permet d'analyser une plage spectrale de l'ordre de $400 \AA$. ii) La résolution optimale est de $0,035 \AA$. Dans la figure 1 , nous donnons les enregistrements de raies d'émission du mercure (4358 $\AA$ et $5461 \AA$ ) obtenus avec des fentes de largeur $10 \mu \mathrm{m}$. Ces enregistrements montrent que la résolution est comprise entre 0,03 et $0,04 \AA$. Comme il est difficile d'utiliser en pratique des fentes moindres que $10 \mu \mathrm{m}$, la résolution optimale est comprise entre ces deux valeurs. Elle est du même ordre que celle du spectrographe.
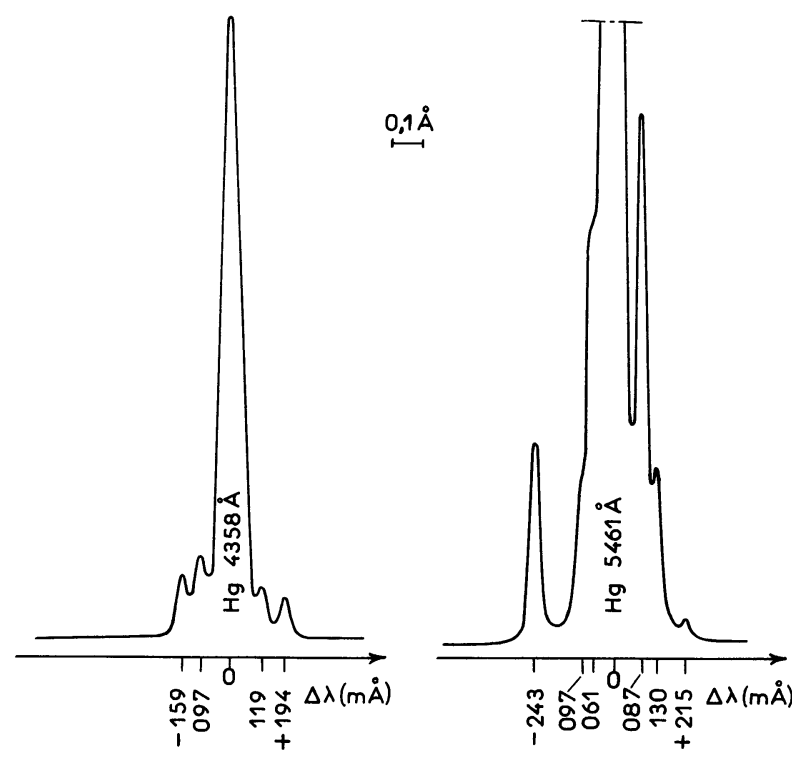

Fig. 1. - Enregistrement photoélectrique de raies d'émission du Mercure. Les fentes ont une largeur de $10 \mu \mathrm{m}$.

[Photoelectric recording of emission lines of Mercury. The width of the slits is $10 \mu \mathrm{m}$. 
iii) La reproductibilité du repérage (relatif) des longueurs d'onde est de $0,01 \AA$. Cette valeur, obtenue après de multiples enregistrements d'une même raie, est nettement meilleure que celle de la plupart des monochromateurs. Dans ces derniers, le repérage est associé à la rotation du réseau. Pour un monochromateur disposant d'un réseau et d'une distance focale identiques à ceux du spectrographe, un défilement de $0,1 \AA$, au voisinage de $4000 \AA$, correspond à une rotation de $10^{-5}$ radians soit $2^{\prime \prime}$ d'angle. Rappelons que dans notre cas, un défilement de $0,1 \AA$ correspond à une rotation de $1 / 20$ de tour de la vis soit $18^{\circ}$. La comparaison de ces deux rotations montre pourquoi, malgré son aspect rudimentaire, la présente détection dispose d'un repérage aussi précis.

Les performances de cette adaptation en font un outil de choix pour l'étude des spectres d'échantillons soumis à des perturbations extérieures. En particulier, l'excellent repérage permet de mesurer les faibles déplacements des structures du spectre en fonction de la perturbation.

$\mathrm{La}$ détection photoélectrique nous a permis entre autres l'emploi de méthodes de modulation [1-5]. Parmi celles qui peuvent être extérieures à l'appareil dispersif, citons le cas de la lumière dont on module l'état de polarisation $[1,2,3]$ ou la modulation spatiale, utilisée pour comparer deux échantillons [4]. Nous avons également équipé notre appareil dispersif d'un dispositif pour moduler la longueur d'onde. Cette modulation est obtenue au moyen d'une lame de quartz oscillante placée juste derrière la fente d'entrée du spectrographe. Sa fréquence de modulation est de quelques centaines de hertz avec une amplitude de modulation pouvant atteindre $0,3 \AA$ [5].

$\mathrm{La}$ figure 2 illustre un cas où la détection photographique doit être associée à une détection photoélectrique. Il s'agit de photographies du spectre de réflexion de la raie $v_{2 \mathrm{~d}}(4062 \AA)$ de $\mathrm{CuBr}$ à 4,2 K. Dans le cas $a$, l'échantillon est libre tandis que dans les cas $b$ et $c$, il est soumis à une pression uniaxiale parallèle à un axe [001] du cristal. Ceci provoque soit un déplacement soit une décomposition de la raie, suivant que la polarisation est parallèle (cas $c$ ) ou perpendiculaire (cas $b$ ) à la pression. L'inclinaison que l'on note sur la figure traduit sans ambiguité l'existence d'un important gradient de pression dans l'échantillon. Une analyse photoélectrique directe des spectres, tels que

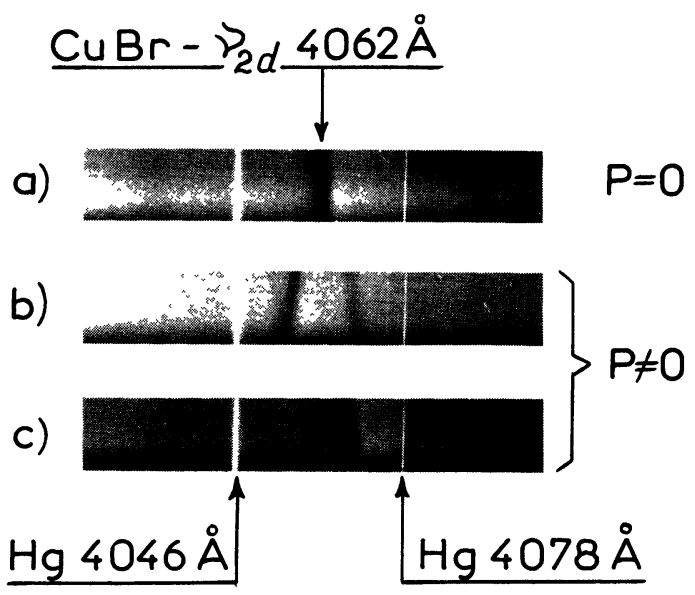

Fig. 2. - Structure d'un spectre perturbé par une pression non uniforme. La hauteur des spectres correspond à $1 \mathrm{~mm}$ au niveau de l'échantillon.

[Structure of a spectrum perturbed by a nonuniform stress. The height of the spectra corresponds to $1 \mathrm{~mm}$ of the sample.]

ceux des figures $2 b$ et $2 c$, aurait conduit à des résultats erronés. L'association des deux modes de détection comme ci-dessus est avantageuse dans d'autres cas, en particulier pour détecter des gradients thermiques [3] ou des inhomogénéités dues par exemple au clivage des cristaux lamellaires.

En conclusion, une adaptation, telle que celle que nous avons réalisée, est possible sur la plupart des spectrographes existants. Elle a l'avantage d'être particulièrement simple et permet d'obtenir des performances comparables à celles des monochromateurs de mêmes caractéristiques. Le mécanisme de défilement étant extérieur à l'appareil dispersif, il peut être facilement adapté aux besoins spécifiques de l'expérience (défilement asservi par exemple). Son entretien et son contrôle ou même son changement en cas d'usure sont aisés. L'aspect modulaire d'un tel spectrophotomètre lui confère une longévité importante, car les spectrographes ne comportent que très peu de pièces mobiles sujettes à usure mécanique. De plus, en raison de la bonne reproductibilité du repérage des longueurs d'onde, cet ensemble peut être plus performant qu'un monochromateur pour certaines applications.

\section{Bibliographie}

[1] Daunois, A., Deiss, J. L., Merle, J. C., Wecker, C., NikiTINE, S., Proc. of XIth Int. Conf. on Phys. of Semiconductors, Varsovie (1972) 1402.

[2] Merle, J. C., Wecker, C., Daunois, A., Deiss, J. L., Nik ITINE, S., Surf. Sci. 37 (1973) 347.

[3] Merle, J. C., Nikitine, S., Haken, H., Phys. Status Solidi (b) 61 (1974) 229.
[4] GIEs, J. G. (à paraître).

[5] Merle, J. C., Wecker, C., Fiorini, P., Proc. of XIVth Int. Conf. on Phys. of Semiconductors, Edimbourg (1978) 1093. 